\title{
Transition form factor of the ${ }^{4} \mathrm{He}\left(\mathrm{O}_{+}\right)$monopole resonance
}

\author{
Simon Kegel* on behalf of the A1 collaboration \\ Institut für Kernphysik der Universität Mainz \\ Johann-Joachim-Becher-Weg 45 \\ 55128 Mainz \\ Germany \\ E-mail: kegel@kph.uni-mainz.de
}

\begin{abstract}
Electron-scattering-experiments offer an insight into nuclear structure and the involved nuclear forces. During the last years, a lot of effort and progress was made by including 3- and 4-body terms in nuclear potentials to gain better results for physical observables by ab-initio calculations. The transition form factor of the ${ }^{4} \mathrm{He}(0+)$-resonance obtained by this new calculations is in significant disagreement with the existing ab-initio calculations to commonly used potentials.

Using Mainz data from 2009 in the $q^{2}$ range from 0.5 to $6.8 \mathrm{fm}^{-2}$, new precise values of the transition form factor will be obtained in order to forward different calculations and better understand the nuclear interactions in the low-momentum-transfer regime.

In this contribution we present the challenges of the data analysis which are related mostly to the determination of the luminosity and subtraction of the background, underlying the monopole resonance of ${ }^{4} \mathrm{He}$ at $20.21 \mathrm{MeV}$ at the threshold of the continuum.
\end{abstract}

54th International Winter Meeting on Nuclear Physics

25-29 January 2016

Bormio, Italy

\footnotetext{
*Speaker.
} 


\section{Motivation}

The alpha particle as a light, but strongly bound system of four nucleons serves as a playground for testing new potentials and different ab-initio calculations using effective-field-theory (EFT). ${ }^{4} \mathrm{He}$ is the lightest nucleus with a resonance at the energy of $20.21 \mathrm{MeV}$ [5].

Next to the properties of the ground state, the characteristics of its resonance represent a stringent test of the calculations. Since the wave function of the resonance is orthogonal to the wave function of the ground state, we expect a quite different spatial structure between those two states. The transition form factor is in impulse approximation the Fourier transform of the transition probability $\rho_{0_{2}^{+} 0_{1}^{+}}(\vec{r}) Y_{00}(\vec{r})$ between ground state $0_{1}^{+}$and resonance $0_{2}^{+}$:

$$
\left.\left|F_{\text {mono }}\left(q^{2}\right)\right|^{2} \propto \frac{1}{4 \pi} \mid \int e^{i \vec{q} \cdot \vec{r}} \rho_{0_{2}^{+} 0_{1}^{+}}(\vec{r}) Y_{00}(\vec{r})\right)\left.d \vec{r}\right|^{2}
$$

Fig. 1 shows different plots of the transition form factor $F_{\text {mono }}\left(q^{2}\right)$ for several ab-initio calculations.

An approach from Hiyama et al. [2] using 3-nucleon-forces on phenomenological basis is matching the data well, also like the result of the phenomenological AV18+UIX potential. The transition form factor originating from EFT-potentials including 3-body-forces up to $\left(N^{2} L O\right)$ by Bacca et al. [1] differs strongly from existing data, though the large error bars (Walcher et al. [5], Köbschall et al. [3], Frosch et al. [4] ) leave remaining doubts for the theory.

The electron accelerator MAMI in Mainz and the A1 collaboration will provide new data with higher statistics to clear the present circumstances.

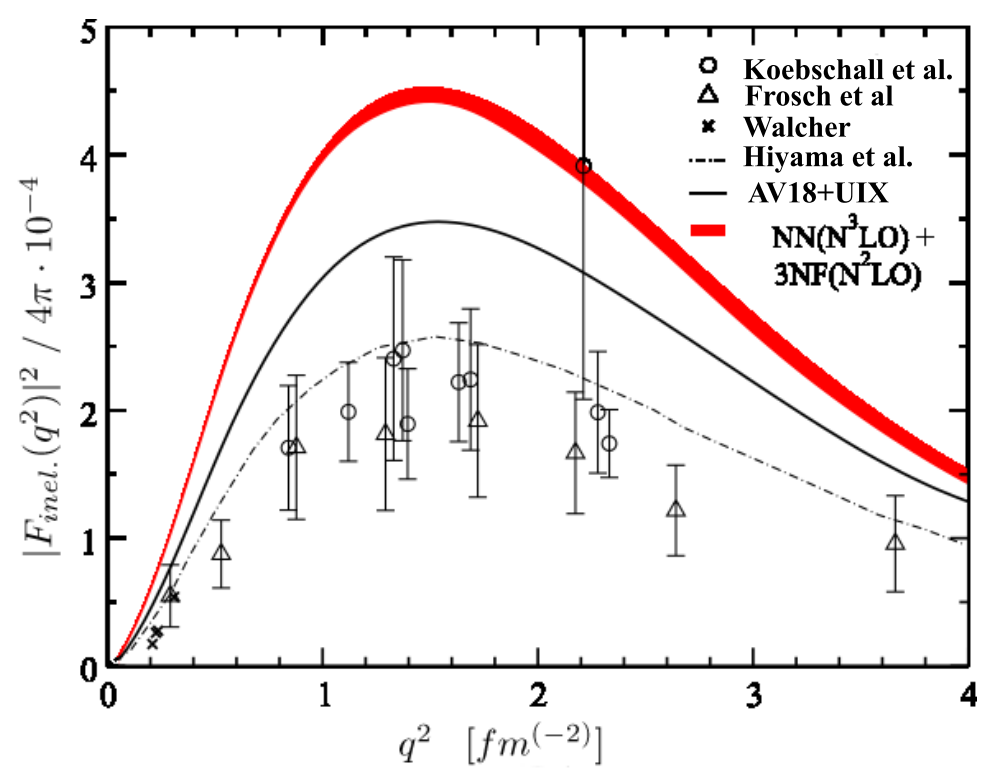

Figure 1: Transition form factor of the monopole resonance of ${ }^{4} \mathrm{He}$ calculated with different nuclear potentials in comparison with existing data [1]. 


\section{Experimental Setup}

\subsection{Mainz Microtron}

The MAMI (Mainz Microtron) electron accelerator is a continuous wave race-track-microtron, providing beam intensities up to $100 \mu \mathrm{A}$ and is thus ideal for experiments which profit from high beam quality. The energy of the electrons can be tuned in $15 \mathrm{MeV}$ steps from $180 \mathrm{MeV}$ to $1.6 \mathrm{GeV}$. After passing the stages of the electron accelerator, the electrons can be guided to the experimental halls. Our experiment was performed in the spectrometer hall of A1 dedicated to precise measurements of form factors. A floor plan of the whole accelerator and detector hall installation is shown in fig. 2.
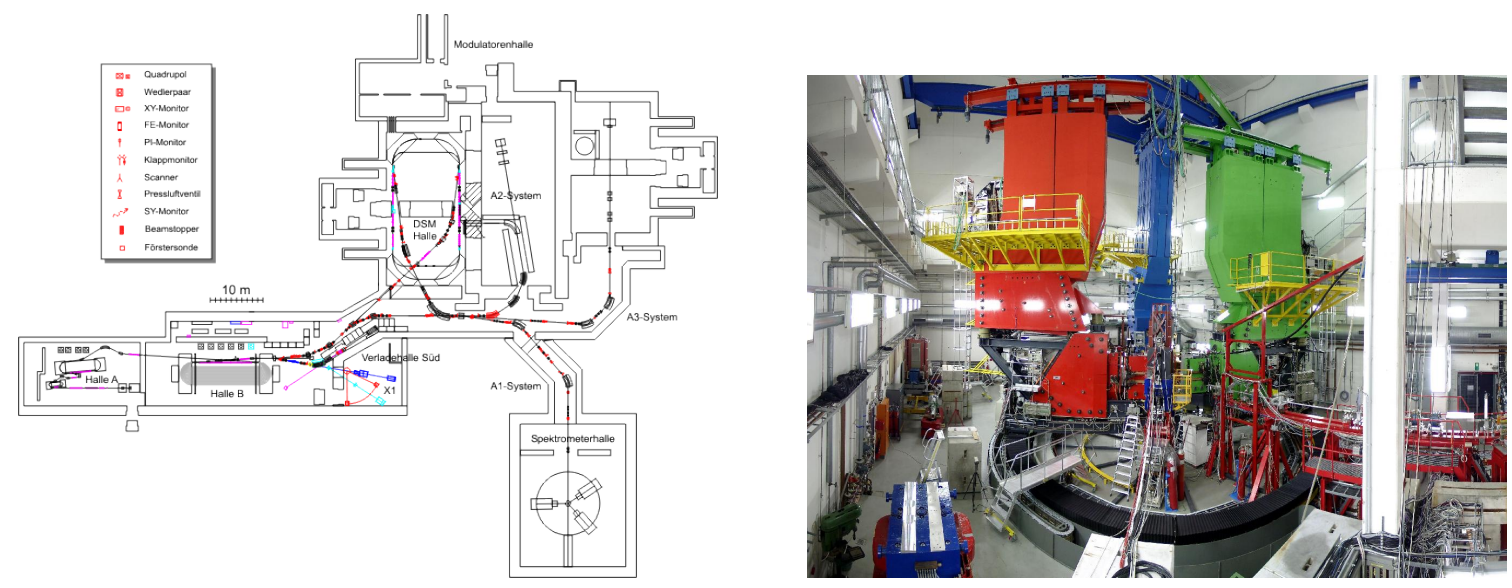

Figure 2: Left: Floor plan of the MAMI accelerator facility in Mainz. Right: View into the detector hall of the A1 collaboration with the three high-resolution magnetic spectrometers.

\subsection{Spectrometer System}

The A1 detection system consists of three high-resolution magnetic spectrometers rotatable around the target over a wide range of possible scattering angles. Vertical and horizontal drift chambers are capable of tracking the particles, guided to the focal plane of the spectrometer by its magnets. Different layers of scintillators are used for triggering. A gas-Cherenkov-detector can identify and distinguish electrons from other particles. The so arranged ensemble of detectors can attain a relative momentum resolution of $\delta p / p=10^{-4}$. A schematic structuring of spectrometer A is shown in fig. 3 .

\subsection{Helium target}

After being guided to the experimental hall, the electrons hit the cryogenic target filled with helium. With a target cell shaped of a single aluminum block, one has the advantage of thin walls of about $250 \mu \mathrm{m}$ to reduce background to a minimum, without loosing stability of the cell, which has to defy gas pressures of over 20 bar. A cooling system with liquid hydrogen as heat exchange medium was capable of cooling down the target gas below temperatures of $20 \mathrm{~K}$. Thus, gas densities of $50 \mathrm{mg} / \mathrm{cm}^{3}$ were reached in the target cell, yielding luminosities during the experiment in the order of $10^{9} \mu \mathrm{b}^{-1}$. A sketch of the target is shown in fig. 3 . 

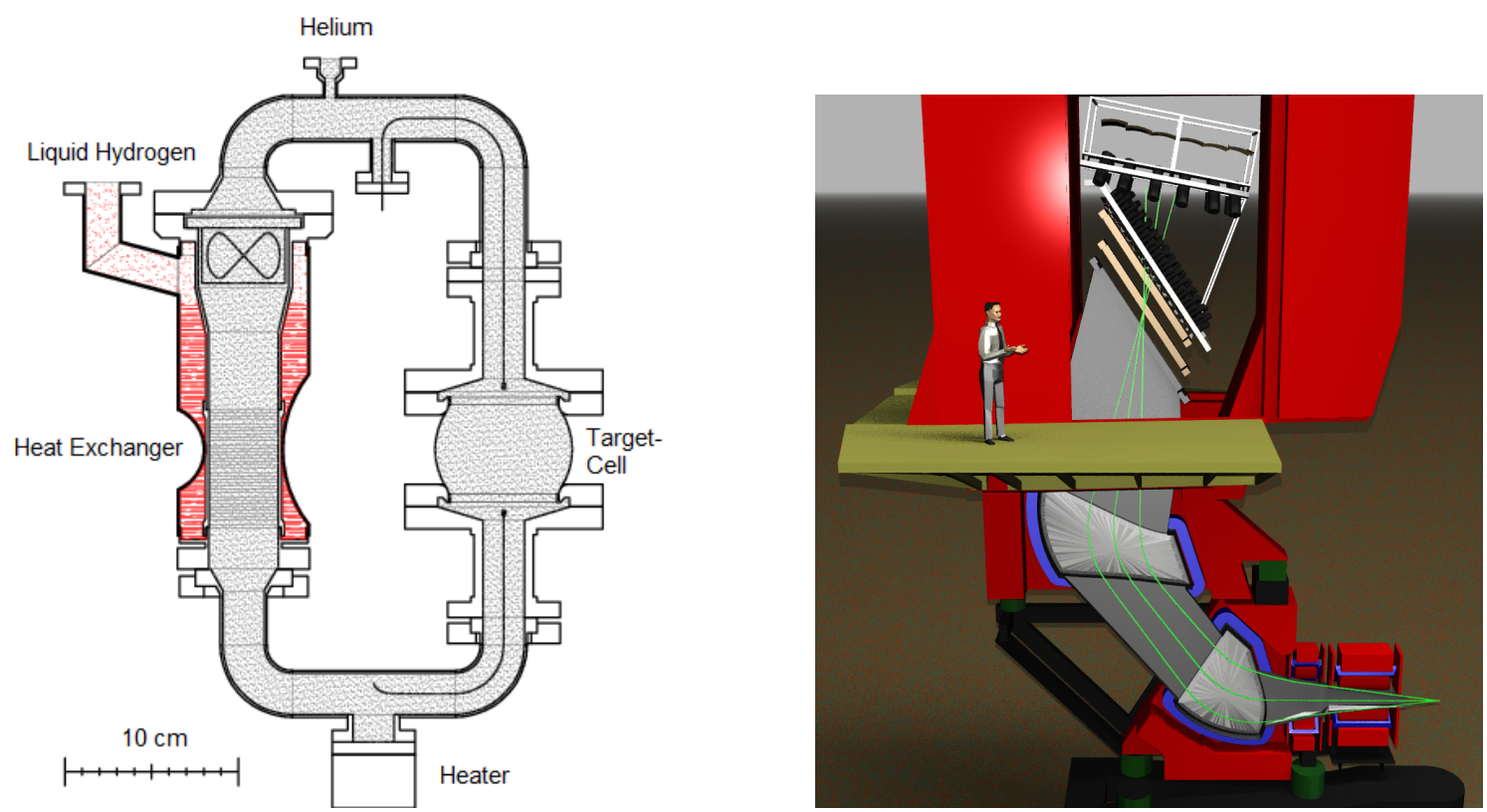

Figure 3: Left: Sketch of the cryogenic helium target, used during the beam time of 2009 in the A1 collaboration. Right: View into the housing of spectrometer A.

\subsection{Experimental Procedure}

Typically, the form factor experiments performed in A1 use more than one spectrometer. With given energy of the electron beam, the spectrometers were arranged with different angles to efficiently scan the kinematic region which is needed. The beam current can therefore go from few nanoamperes to $20 \mu \mathrm{A}$ for cryogenic gas targets up to $100 \mu \mathrm{A}$ for solid state targets like tantalum, that can stand the high heat dissipation by the electron beam. To avoid a large difference in the trigger rate of two spectrometers being set at different angles due to the sharp drop of the cross section at higher scattering angles, the trigger rate of each spectrometer can be adjusted by a prescaler. Thus, sufficient statistics is reached with count rates in the order of $100 \mathrm{~Hz}$.

This experiment was covering energies from $180 \mathrm{MeV}$ to $720 \mathrm{MeV}$ in steps of $135 \mathrm{MeV}$. The scattering angles were chosen in that way, that the $q^{2}$ values are reproduced in the different energy settings. The advantage for the experiment is thus a maximum of redundancy.

\section{Data Analysis}

The goal of the analysis is to extract events, descending from the ${ }^{4} \mathrm{He}(0+)$ resonance peak and to determine the transition form factor.

Describing the monopole resonance in Breit-Wigner-frame, the monopole peak can in terms of missing mass be represented as a Lorentz-curve, with a width of $\Gamma_{\text {exp }}=270 \pm 50 \mathrm{keV}$, located at 20.21 MeV [5]. Due to the fact that the peak is at the beginning of the break-up continuum, the extraction of the resonance regards a detailed subtraction of background. It turns out that at the 
threshold the continuum can be well described by the potential functions $x^{n}$, where $n<1$. Hence, we used this ansatz together with the Lorentz peak function to fit the data:

$$
f_{\text {res }}\left(x, A, \Gamma, x_{0}, m, n, c\right)=\frac{1}{\pi} \frac{A \cdot \Gamma}{\left(x-x_{0}\right)^{2}+\frac{\Gamma^{2}}{4}}+\Theta\left(x-\left(x_{0}-\delta\right)\right) \cdot m\left(x-\left(x_{0}-\delta\right)\right)^{n}+c
$$

The parameter $m$ serves as a scaling ratio of the events in the quasi-elastic region, $\delta$ is the difference between peak center and beginning of the continuum in $\mathrm{MeV}$, which can in this way be considered as a constraint. The Heaviside step-function makes sure that the continuum is not contributing before the threshold of $19.85 \mathrm{MeV}$.

With regard to the low count rates of the resonance, we decided to use the Maximum-LikelihoodFit method, that already includes the Poisson-statistics of the bins in the missing mass histogram. The function to minimize for the best fit curve is thus:

$$
\mathscr{L}(x, n, \vec{p})=-\sum_{i} n_{i} \ln \left(f\left(x_{i}, \vec{p}\right)\right)-f\left(x_{i}, \vec{p}\right)-\ln \left(n_{i} !\right)
$$

Here, $n_{i}$ is the content of bin i, $\vec{p}$ is a short-hand notation for all parameters of the fit function $f(x, \vec{p})$. The result of an example fit with parameters $A, \Gamma, x_{0}, \delta, m, n, c$ is shown in fig. 4.

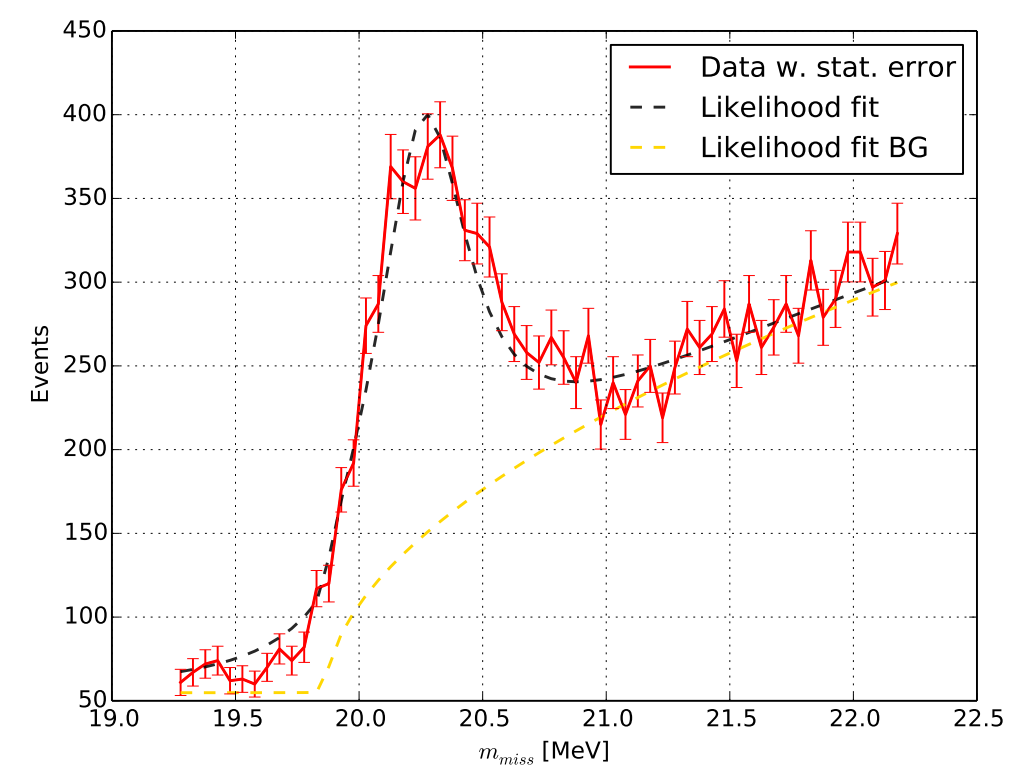

Figure 4: Set of data with statistical error, showing the missing mass spectrum in the region of the ${ }^{4} \mathrm{He}(0+)$ resonance at $20.21 \mathrm{MeV}$.

With the content of the bins of the resonance peak in the histogram, related to scattered electrons, it is possible to determine the form factor as ratio between experimental cross section and Mott-cross-section: 


$$
\left|F_{\text {mono }}\left(q^{2}\right)\right|^{2}=\left(\frac{d \sigma}{d \Omega}\right)_{\text {exp }} / 4 \pi\left(\frac{d \sigma}{d \Omega}\right)_{M o t t}
$$

In this step, we need to consider the dependency of the measured cross section on the luminosity, which is in the case of using a gaseous target very susceptible to fluctuations of temperature and density. The experiment was designed in a way, that for the configuration of fixed $q^{2}$, a setup with the elastic line of the ground state was included in the acceptance. Moving to another setup of different angle and momentum transfer, one can use the overlapping part of setups and precise knowledge of the elastic form factor to compare and adjust luminosity fluctuations. Fig. 5 shows the scanned kinematic region of constant energy with lines of constant $q^{2}$ and $\vec{q}^{2}$ by varying angle and central momentum of the spectrometer. The size of the rectangular patches illustrates the angular and momentum acceptance of the setup. The experiment was designed in that way, that when moving on lines of constant $q^{2}$, there is always an area where two or more setups overlap in the kinematic diagram.

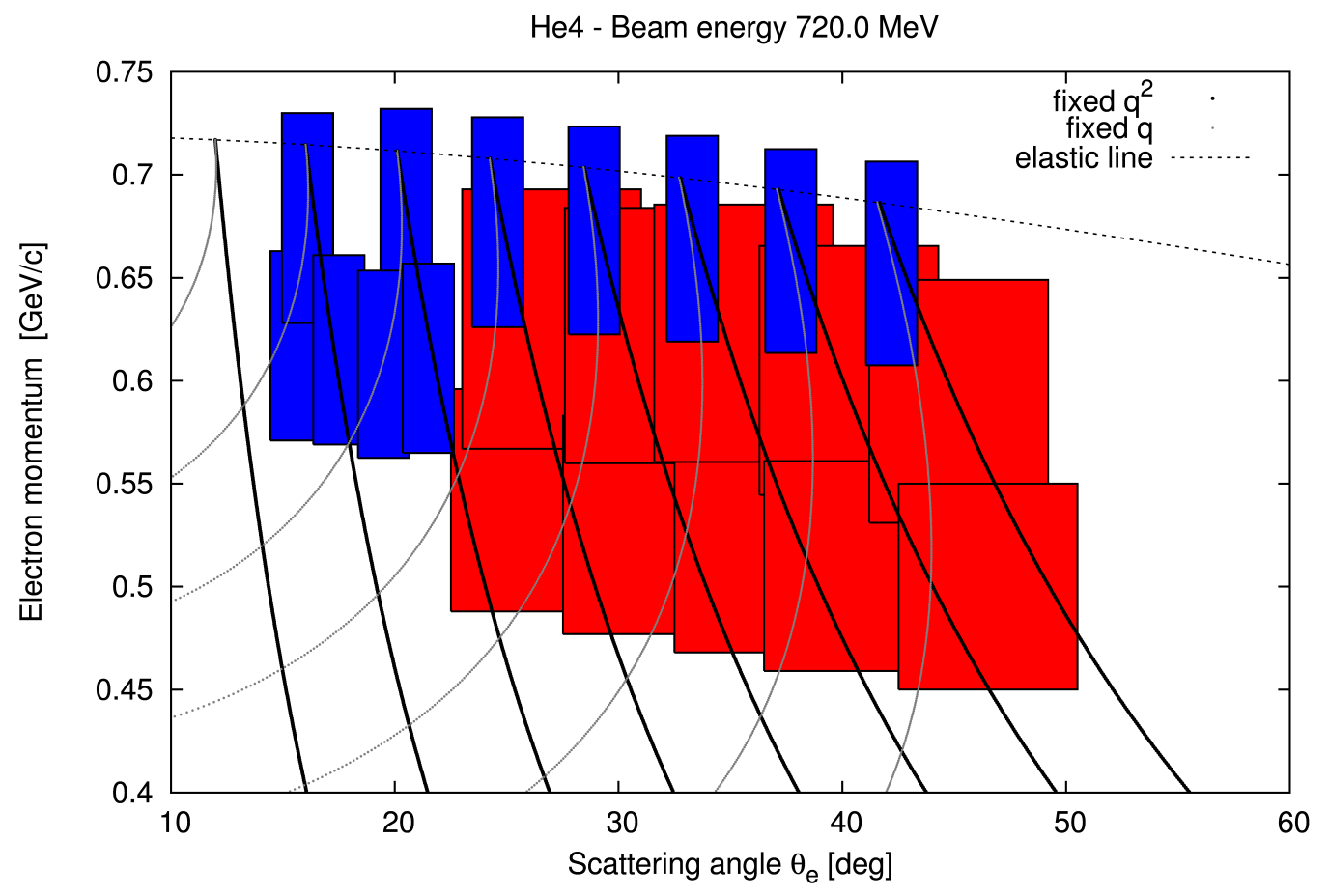

Figure 5: Kinematic region for beam energy $720 \mathrm{MeV}$. The dashed upper line indicates the position of the elastic line of ${ }^{4} \mathrm{He}$, while the black and gray solid lines indicate constant values of $q^{2}$ and $\vec{q}^{2}$ respectively. The covered area by the red patches is related to the angular and momentum acceptance of spectrometer A in the kinematic diagram, while the blue patches belong to the acceptance region of spectrometer B.

Preliminary results of data points obtained by this method are shown in fig. 6 . The data points are from setups with an elastic line included, to be aware of errors arising from luminosity or acceptance. Error bars are not included in this plot because the error estimation has not yet been completed. 


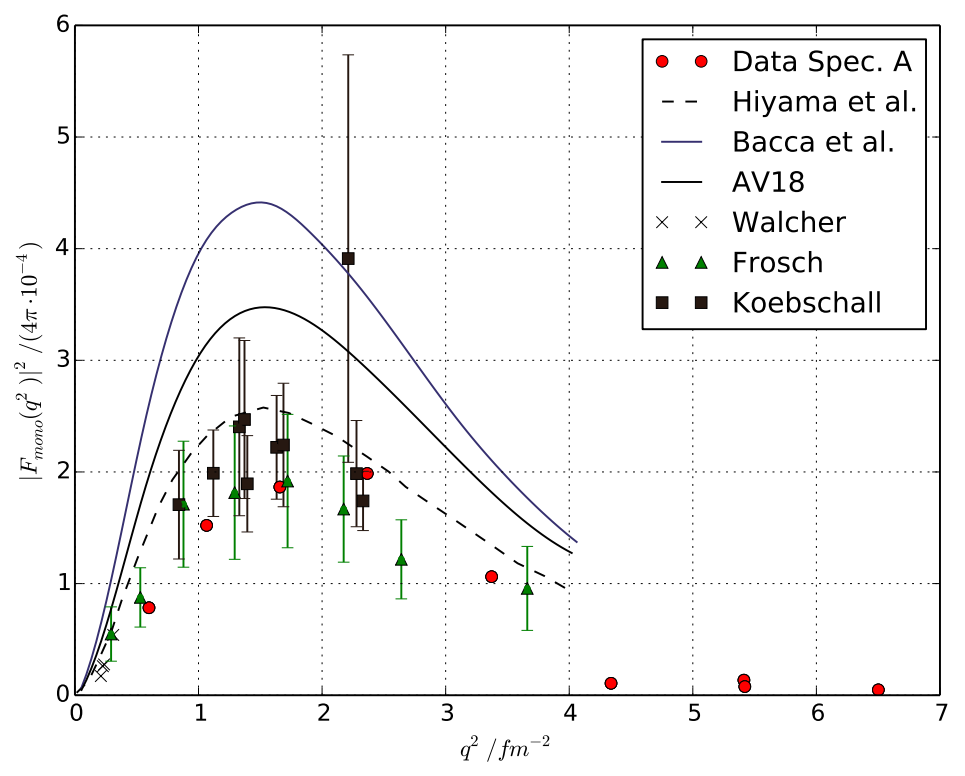

Figure 6: Preliminary results of the resonance form factor $\left|F_{\text {mono }}\left(q^{2}\right)\right|$. Data points and theoretical curves were adopted from [1].

\section{Summary and Outlook}

Here, an experiment was presented, aiming to determine the transition form factor $F_{\text {res }}\left(q^{2}\right)$ over a wide range of $q^{2}$. With the ongoing work we want to inspire the discussion and improvement of nuclear potentials, EFT and ab-initio calculations.

To complement the existing data, another electron scattering experiment is scheduled for 2016, which will employ an improved helium target with better temperature and pressure control. The kinematics to be measured in the upcoming experiment will be associated to the former experiment. 


\section{References}

[1] Isoscalar Monopole Resonance of the Alpha Particle: A Prism to Nuclear Hamiltonians S. Bacca, N. Barnea, W. Leidemann and G. Orlandini, Physical Review Letter 110, 042503 (2013)

[2] Four-body calculation of the first excited state of ${ }^{4} \mathrm{He}$ using a realistic NN interaction: ${ }^{4} \mathrm{He}\left(e, e^{\prime}\right)^{4} \mathrm{He}\left(0^{+}\right)$and the monopole sum rule

E. Hiyami, B. F. Gibson and M. Kamimura, Physical Review 70, 031001(R) (2004)

[3] Excitation of the quasi-bound state in ${ }^{4}$ He by electron scattering at medium momentum transfer G. Köbschall, C. Ottermann, K. Maurer, K. Röhrich, Ch. Schmitt and V. H. Walther, Nuclear Physics A405 (1983) 648-652

[4] Inelastic electron scattering from ${ }^{4} \mathrm{He}$

R. F. Frosch, R. E. Rand and H. L. Cranell, J. S. McCarthy, L. R. Suelzle and M. R. Yearian, Nuclear Physics A110, 657 (1968)

[5] Unelastische Elektronenstreuung am ${ }^{4}$ He bei kleinem Impulsübertrag*

Th. Walcher, Z. Physik 237, 368-380 (1970) 